\title{
A High-Throughput Random Access Protocol for Multiuser MIMO Systems
}

\author{
Haiyou Guo, ${ }^{1}$ Honglin Hu, ${ }^{1}$ and Yan Zhang ${ }^{2}$ \\ ${ }^{1}$ Shanghai Research Center for Wireless Communications, Shanghai Institute of Microsystem and Information Technology, \\ Shanghai 200050, China \\ ${ }^{2}$ Simula Research Laboratory, 1325 Lysaker, Norway
}

Correspondence should be addressed to Honglin Hu, hlhu@ieee.org

Received 14 March 2008; Accepted 28 April 2008

Recommended by Edward K. S. Au

We propose a high-throughput random access protocol for $2 \times 2$ multiuser multiple-input multiple-output (MIMO) systems. The cross-layer mechanism utilizes the packets combining technique to exploit the advantages of both spatial multiplexing and multipacket reception. Analytical result indicates that the proposed scheme achieves 0.669 per spatial degree of freedom in stable throughput, which is much higher than those in the existed studies.

Copyright (C) 2008 Haiyou Guo et al. This is an open access article distributed under the Creative Commons Attribution License, which permits unrestricted use, distribution, and reproduction in any medium, provided the original work is properly cited.

\section{Introduction}

Random access (RA) is a cost-efficient medium access process in next generation wireless networks to support highrate high-capacity multimedia services. The traditional RA schemes, for example, slotted ALOHA, incur the throughput penalty due to serious collision. The emerging multipleinput multiple-output (MIMO) technology provides a new dimension of performance enhancement for RA schemes. MIMO is able to improve the throughput in two aspects, that is, a single-user throughput improvement via the spatial multiplexing (SM) such as V-BLAST [1]; and the whole system throughput improvement via the multipacket reception (MPR) capability [2].

Collision resolution (CR) has been investigated in both MIMO systems and single-antenna systems. For MIMO systems, a multiuser detector was developed with spacetime signature induced by the Alamouti-type space-time block code (STBC) [2]. Consequently, the slotted ALOHA can operate over Alamouti-type STBC to achieve the MPR capability. We will term this scheme as ALOHA-Alamouti. In the $2 \times 2$ multiuser MIMO system, however, such MPR is at most applicable for the 2-fold collision, and hence incurs the undesirable throughput loss for the moments of severe collision more than 2 folds due to the ineffective Alamouti- type coding. For the single-antenna systems, multiuser packets combining techniques were employed in network diversity multiple access (NDMA) [3] and successive interference cancellation tree algorithm (SICTA) [4] to improve the throughput. It has been shown that SICTA has the highest throughput 0.693 among the splitting algorithms. However, SICTA requires unbounded amount of memory to store the mixed packets, and NDMA may be inapplicable in multiuser MIMO due to the assumption of the fading channel coherence time in the order of the packet slot. This assumption may not be realistic since the channel coefficients are usually correlated over adjacent slots.

In this letter, we propose a high-throughput RA protocol for $2 \times 2$ multiuser MIMO systems. With a cross-layer design approach, our scheme utilizes the adaptive packets combining technique to reconstruct Alamouti-type STBC only for the 2-fold collision, and hence avoids the undesirable throughput loss. Then, the scheme can exploit the benefits of both V-BLAST and Alamouti-type STBC to improve the throughput. The proposed scheme is able to achieve stable throughput as high as 0.669 per spatial degree of freedom while maintaining the implementation simplicity like slotted ALOHA. In addition, the scheme can operate on the block fading channel without the constraint on the coherence time. 


\section{Notations}

Bold uppercase letters denote matrices and bold lowercase letters denote vectors. Complex conjugate, transpose, and inverse operations are represented by $(\cdot)^{*},(\cdot)^{T}$, and $(\cdot)^{-1}$, respectively. $[\mathbf{A}]_{i j}$ denotes the $(i, j)$ entry of matrix $\mathbf{A} . \mathbf{I}_{M}$ is the identity matrix of size $M .|\cdot|$ represents the cardinality of a set.

\section{Modeling Preliminaries}

In a cellular network, there is a set of users, denoted as $Q=\{1,2, \ldots, q, \ldots\}$, communicating with the base station (BS). All transmissions initiated by a source $q$ are directed to a single destination $d \notin Q$. Each user and the BS are equipped with two antennas. The system is equally slotted in time, and the length of one time slot is $T$.

At each slot $n$, each user $q$ may transmit a packet consisting of $2 L$ symbols, which can be demultiplexed into two subpackets with the same length and fed into two transmit antennas. We denote these subpackets as

$$
\begin{aligned}
& \mathbf{x}_{q}(n)=\left[\begin{array}{llll}
x_{n, q}(1) & x_{n, q}(2) & \cdots & x_{n, q}(L)
\end{array}\right] \\
& \mathbf{y}_{q}(n)=\left[\begin{array}{llll}
y_{n, q}(1) & y_{n, q}(2) & \cdots & y_{n, q}(L)
\end{array}\right] .
\end{aligned}
$$

The symbols $x_{n, q}(l)$ and $y_{n, q}(l), l=1,2, \ldots, L$, are assumed drawn from a finite QAM constellation. We assume the symbol period is $\tau$ such that $T=L \tau$. Let $I(n) \subseteq Q$ denote the set of users who are active at slot $n$. If $|I(n)| \geq 2$, we say that an $|I(n)|$-fold collision occurs in the channel.

In our proposed scheme as shown in Figure 1, we define three exclusive categories of time slot: remediable colliding slot (RC-Slot), compensating slot (C-Slot), and trivial slot (T-Slot). If and only if $|I(n)|=2$ and $|I(n-1)| \neq 2$, we refer to slot $n$ and $n+1$ as the RC-Slot and the C-Slot, respectively. The $C$-Slot immediately follows the RC-Slot; and it is reserved to retransmit the contended packets during the RC-Slot. A slot is termed as T-Slot in case it is neither the RC-Slot nor the $C$-Slot. Each slot serves as only one of the three types. In Figure 1, slot $n$ and $n+3$ are RC-Slot; slot $n+1$ and $n+4$ are $C$-Slot; and the other slots are $T$-Slot.

If slot $n$ acts as a $T$-Slot or an RC-Slot, the active user transmits/retransmits the packets in the original form of (1). The received baseband discrete-time signal at the BS is

$$
\mathbf{z}_{n}(l)=\sum_{q \in I(n)} \mathbf{H}_{n, q}(l)\left[\begin{array}{l}
x_{n, q}(l) \\
y_{n, q}(l)
\end{array}\right]+\mathbf{v}_{n}(l), \quad l=1,2, \ldots, L,
$$

where $\mathbf{v}_{n}(l)$ is additive noise; and $\mathbf{H}_{n, q}(l)$ is the $q$ th user's $2 \times$ 2 channel matrix. On the other hand, if slot $n$ acts as a $C$ Slot, only the two users attributed to $I(n-1)$ are allowed to retransmit their packets in the compensating form of $\mathbf{x}_{q}^{*}(n-$ $1)$ and $-y_{q}^{*}(n-1)$. Specifically, the received signal at the $C$ Slot $n$ can be written as

$$
\mathbf{z}_{n}(l)=\sum_{q \in I(n-1)} \mathbf{H}_{n, q}(l)\left[\begin{array}{c}
-y_{n-1, q}^{*}(l) \\
x_{n-1, q}^{*}(l)
\end{array}\right]+\mathbf{v}_{n}(l), \quad l=1,2, \ldots, L .
$$

We assume a flat fading channel and the channel matrices are independent across different users. The BS has the perfect channel state information. These assumptions are reasonable in the pilot-assisted orthogonal frequency division multiplexing (OFDM) systems. All the entries of $\mathbf{H}_{n, q}(l)$ are with zero mean, independent real, and imaginary parts, forming an i.i.d. Gaussian collection. Note that the channel coefficients do not change significantly during the period of $(L+1) \tau$ to meet the requirement of correct demodulation for the reconstructed Alamouti codes, which indicates

$$
\mathbf{H}_{n-1, q}(l)=\mathbf{H}_{n, q}(l), \quad l=1,2, \ldots, L .
$$

\section{High-throughput RA Protocol for $2 \times 2$ Multiuser MIMO systems}

In the proposed scheme, the V-BLAST is designated as a basic mode while the Alamouti-type STBC can be reconstructed by the packets combining for the remediable 2 -fold collision.

\subsection{Descriptions of Proposed Protocol}

The mechanism of the proposed protocol is specified as follows.

\subsubsection{Immediate Feedback}

At the end of each slot $n$, each user obtains a 4-ary feedback from the BS with the information of $|I(n)|$. Such a feedback indicates the information of $|I(n)|=0,|I(n)|=1,|I(n)|=$ 2 , and $|I(n)|>2$. Based on the feedback and its own state, each user can deduce the category of the current slot and decide the operation in the next slot. To determine the number of the users involved in the collision, the appropriate collision detection technique is employed at the BS similar to that of NDMA [3].

\subsubsection{Initial Transmission}

If one packet arrives during an RC-Slot, its first transmission is randomly scheduled in a slot except the $C$-Slot. Otherwise, it is transmitted immediately at the next slot.

\subsubsection{Collision or Perfect Reception}

If only one user sends a packet in a given slot, the packet is correctly received. If two users send packets during an RCSlot, then the receiver should store the colliding packets; and the next slot is reserved only for these two users as a $C$-Slot. After combining the stored packets with the packets received during the $C$-Slot, the receiver can recover the desired packets from the 2-fold collision. However, no packet information can be extracted from a severe collision more than 2 folds, and the irrecoverable packets are discarded.

\subsubsection{Retransmission of Irremediable Collisions}

Each packet involved in an irremediable collision, independent of the others, must be stochastically retransmitted in 


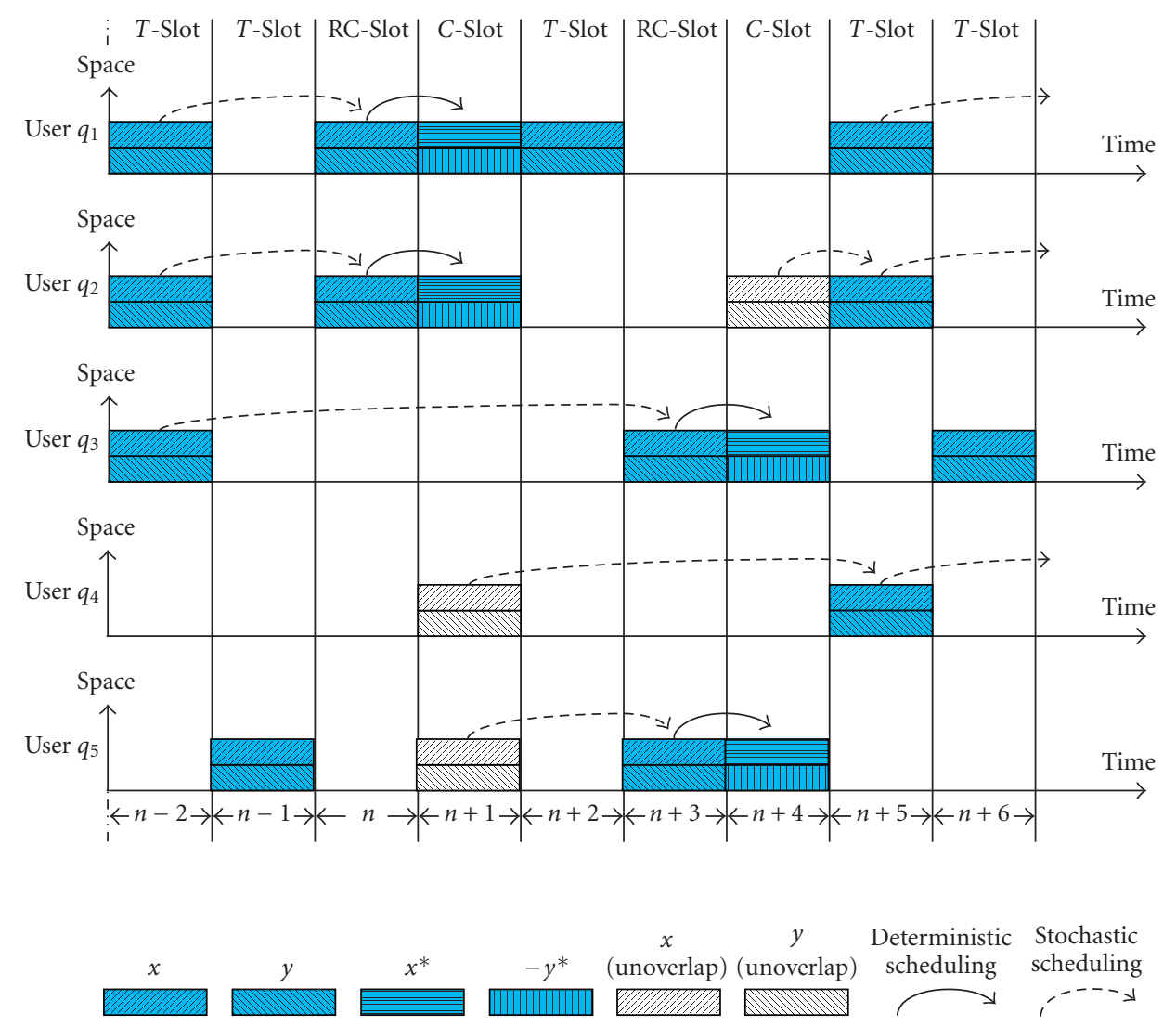

FIGURE 1: Packet scheduling of the high-throughput random access protocol for $2 \times 2$ multiuser MIMO systems.

slots later other than the $C$-Slot until the packet is successfully received.

Figure 1 shows an example of the protocol operation. At the end of slot $n$, since two consecutive feedbacks indicate $|I(n-1)| \neq 2$ and $|I(n)|=2$, the active users during slot $n$, that is, $I(n)=\left\{q_{1}, q_{2}\right\}$, can deduce that slot $n$ and the next slot $n+1$ are RC-Slot and C-Slot, respectively. Thus, the receiver stores the mixed packets in slot $n$; and $\left\{q_{1}, q_{2}\right\}$ will retransmit packets in the compensating form at the immediate next slot. Meanwhile, the inactive users during slot $n$, that is, $\left\{q_{3}, q_{4}, q_{5}\right\}$, can understand the same situation and hence keep silent in the next slot, although $\left\{q_{4}, q_{5}\right\}$ have new arrivals during slot $n$. Thereafter, $\left\{q_{4}, q_{5}\right\}$ will randomly schedule the new arrivals in the future slot except the $C$-Slot to avoid the collision with $\left\{q_{1}, q_{2}\right\}$ in slot $n+1$. In the proposed scheme, each user decides his own operation based on the local information. Particularly, each user behaves independently in the RC-Slot and T-Slot analogous to the slotted ALOHA. The RC-Slot and T-Slot occur stochastically whereas the $C$-Slot succeeds to the RC-Slot determinately.

\subsection{CR through Reconstructed Alamouti-Type STBC}

In the proposed scheme, the colliding packets can be remedied with the help of the Alamouti-type STBC. Let us consider the case in Figure 1 where two users collide in an
RC-Slot $n$. Then, the receiver stores the 2-fold colliding packets during slot $n$, that is, $\mathbf{Z}_{n}=\left[\begin{array}{llll}\mathbf{z}_{n}(1) & \mathbf{z}_{n}(2) & \cdots & \mathbf{z}_{n}(L)\end{array}\right]$, where

$$
\begin{aligned}
\mathbf{z}_{n}(l) & =\left[\begin{array}{l}
z_{n, 1}(l) \\
z_{n, 2}(l)
\end{array}\right] \\
& =\sum_{q \in\left\{q_{1}, q_{2}\right\}} \mathbf{H}_{n, q}(l)\left[\begin{array}{l}
x_{n, q}(l) \\
y_{n, q}(l)
\end{array}\right]+\left[\begin{array}{l}
v_{n, 1}(l) \\
v_{n, 2}(l)
\end{array}\right], \\
l & =1,2, \ldots, L .
\end{aligned}
$$

Once the RC-Slot has been detected, the system immediately enters the $C$-Slot. Thus, the users $\left\{q_{1}, q_{2}\right\}$ send the compensating packet at slot $n+1$. Another 2 -fold colliding packets can be received at the BS, that is, $\mathbf{Z}_{n+1}=$ $\left[\begin{array}{llll}\mathbf{z}_{n+1}(1) & \mathbf{z}_{n+1}(2) & \cdots & \mathbf{z}_{n+1}(L)\end{array}\right]$, where

$$
\begin{aligned}
\mathbf{z}_{n+1}(l) & =\left[\begin{array}{l}
z_{n+1,1}(l) \\
z_{n+1,2}(l)
\end{array}\right] \\
& =\sum_{q \in\left\{q_{1}, q_{2}\right\}} \mathbf{H}_{n+1, q}(l)\left[\begin{array}{c}
-y_{n, q}^{*}(l) \\
x_{n, q}^{*}(l)
\end{array}\right]+\left[\begin{array}{l}
v_{n+1,1}(l) \\
v_{n+1,2}(l)
\end{array}\right], \\
l & =1,2, \ldots, L .
\end{aligned}
$$


Rearranging (5) and (6), with the assumption of (4), we can reconstruct the Alamouti-type STBC. Thus, we have [2]

$$
\begin{array}{r}
\tilde{\mathbf{z}}_{n}(l)=\left[\begin{array}{ll}
\mathbf{H}_{n, q_{1}}(l, 1) & \mathbf{H}_{n, q_{2}}(l, 1) \\
\mathbf{H}_{n, q_{1}}(l, 2) & \mathbf{H}_{n, q_{2}}(l, 2)
\end{array}\right]\left[\begin{array}{l}
\mathbf{a}_{n, q_{1}}(l) \\
\mathbf{a}_{n, q_{2}}(l)
\end{array}\right]+\widetilde{\mathbf{v}}_{n}(l), \\
l=1,2, \ldots, L,
\end{array}
$$

where

$$
\begin{aligned}
& \widetilde{\mathbf{z}}_{n}(l)=\left[\begin{array}{llll}
z_{n, 1}(l) & -z_{n+1,1}^{*}(l) & z_{n, 2}(l) & -z_{n+1,2}^{*}(l)
\end{array}\right]^{T} ; \\
& \widetilde{\mathbf{v}}_{n}(l)=\left[\begin{array}{llll}
v_{n, 1}(l) & v_{n+1,1}(l) & v_{n, 2}(l) & v_{n+1,2}(l)
\end{array}\right]^{T} ; \\
& \mathbf{a}_{n, q}(l)=\left[\begin{array}{ll}
x_{n, q}(l) & y_{n, q}(l)
\end{array}\right]^{T} \text {; } \\
& \mathbf{H}_{n, q}(l, i)=\left[\begin{array}{cc}
{\left[\mathbf{H}_{n, q}(l)\right]_{i, 1}} & {\left[\mathbf{H}_{n, q}(l)\right]_{i, 2}} \\
-\left[\mathbf{H}_{n, q}(l)\right]_{i, 2}^{*} & {\left[\mathbf{H}_{n, q}(l)\right]_{i, 1}^{*}}
\end{array}\right] .
\end{aligned}
$$

By applying the following block linear filter:

$$
\mathbf{W}=\left[\begin{array}{cc}
\mathbf{I}_{2} & -\mathbf{H}_{n, q_{2}}(l, 1) \mathbf{H}_{n, q_{2}}^{-1}(l, 2) \\
-\mathbf{H}_{n, q_{1}}(l, 2) \mathbf{H}_{n, q_{1}}^{-1}(l, 1) & \mathbf{I}_{2}
\end{array}\right]
$$

we extract the original packets from the packet mixture of (7). As a result, we obtain the interference free packets for users $q_{1}$ and $q_{2}$, shown as

$$
\mathbf{W} \widetilde{\mathbf{z}}_{n}(l)=\left[\begin{array}{c}
\widetilde{\mathbf{H}}_{n, q_{1}}(l) \mathbf{a}_{n, q_{1}}(l) \\
\widetilde{\mathbf{H}}_{n, q_{2}}(l) \mathbf{a}_{n, q_{2}}(l)
\end{array}\right]+\mathbf{W} \widetilde{\mathbf{v}}_{n}(l), \quad l=1,2, \ldots, L,
$$

where

$$
\begin{aligned}
\widetilde{\mathbf{H}}_{n, q_{1}}(l) & =\mathbf{H}_{n, q_{1}}(l, 1)-\mathbf{H}_{n, q_{2}}(l, 1) \mathbf{H}_{n, q_{2}}^{-1}(l, 2) \mathbf{H}_{n, q_{1}}(l, 2) ; \\
\widetilde{\mathbf{H}}_{n, q_{2}}(l) & =\mathbf{H}_{n, q_{2}}(l, 2)-\mathbf{H}_{n, q_{1}}(l, 2) \mathbf{H}_{n, q_{1}}^{-1}(l, 1) \mathbf{H}_{n, q_{2}}(l, 1) .
\end{aligned}
$$

Since $\widetilde{\mathbf{H}}_{n, q_{1}}(l)$ and $\widetilde{\mathbf{H}}_{n, q_{2}}(l)$ keep the Alamouti structure [2], the packets involved in the 2-fold collision are recovered with 2 -fold diversity order merely through linear processing. However, to achieve the same diversity gain for the packet without collision, more sophisticated detection algorithm is required other than linear processing. Thus, the multiaccess interference (MAI) can be easily eliminated without sacrificing the space-time diversity gain. Consequently, in the proposed scheme, the transmission reliability is mainly impaired by the severe MAI rather than the noise. In the following throughput analysis, we will consider the error due to packet overlapping rather than that due to the noise.

\subsection{Throughput Analysis}

We evaluate the throughput using the tool similar to that developed for the standard ALOHA under the classical infinite population Poisson model [5, page 48]. Note that for the multiantenna scenario, the throughput is defined as the

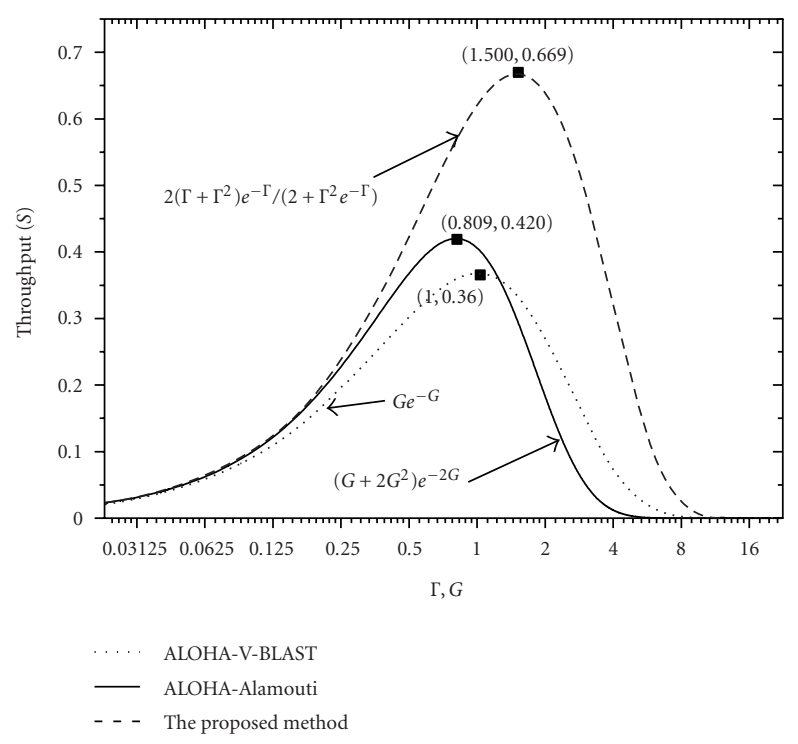

FIgURE 2: Highest throughput per spatial degree of freedom achieved by different random access protocols for $2 \times 2 \mathrm{MIMO}$ systems.

fraction of time during which the space-time channel carries useful information per spatial degree of freedom.

In the proposed scheme, each user behaves independently during the slots except for $C$-Slot. Then, we can assume that the start time of packets in the channel except for $C$-Slot follows a Poisson point process with parameter $\gamma$ packets/second. Let $\Gamma$ denote the normalized intensity, that is, $\Gamma=\gamma T$. During a slot, the average number of the uncolliding subpackets is $2 \times \Gamma e^{-\Gamma}$, and the average number of the remediable colliding subpackets is $4 \times 0.5 \Gamma^{2} e^{-\Gamma}$. Taking into account the extra cost of the $C$-Slot spent on the collision remediation, the average number of the required $C$-Slots is $0.5 \Gamma^{2} e^{-\Gamma}$. Therefore, the throughput, $S$, is given by

$$
S=\frac{2 \Gamma e^{-\Gamma}+2 \Gamma^{2} e^{-\Gamma}}{2\left(1+0.5 \Gamma^{2} e^{-\Gamma}\right)}=\frac{2\left(\Gamma+\Gamma^{2}\right) e^{-\Gamma}}{2+\Gamma^{2} e^{-\Gamma}} .
$$

Figure 2 compares the highest throughput of different RA schemes under the same scenario. Here $G$ is the normalized offered load [5] for ALOHA-Alamouti and ALOHA with V-BLAST. Since the highest throughput is unrelated to the scale of abscissa, we can derive that our proposed scheme, ALOHA-Alamouti, and ALOHA with V-BLAST can reach their own maximal throughput at $0.669,0.420$, and 0.36 per spatial degree of freedom, respectively. The latter two schemes improve the throughput only via MPR or SM, respectively. However, our strategy can permeate the flexibility of Alamouti codes to the MAC layer so as to employ the advantages of both MPR and SM. For the T-Slot without collision, the packet can be exactly received achieving the $\mathrm{SM}$ gain. For the RC-Slot and C-Slot, the packets of two active users can be resolved via the MPR capability of the reconstructed Alamouti-type STBC. Consequently, the 


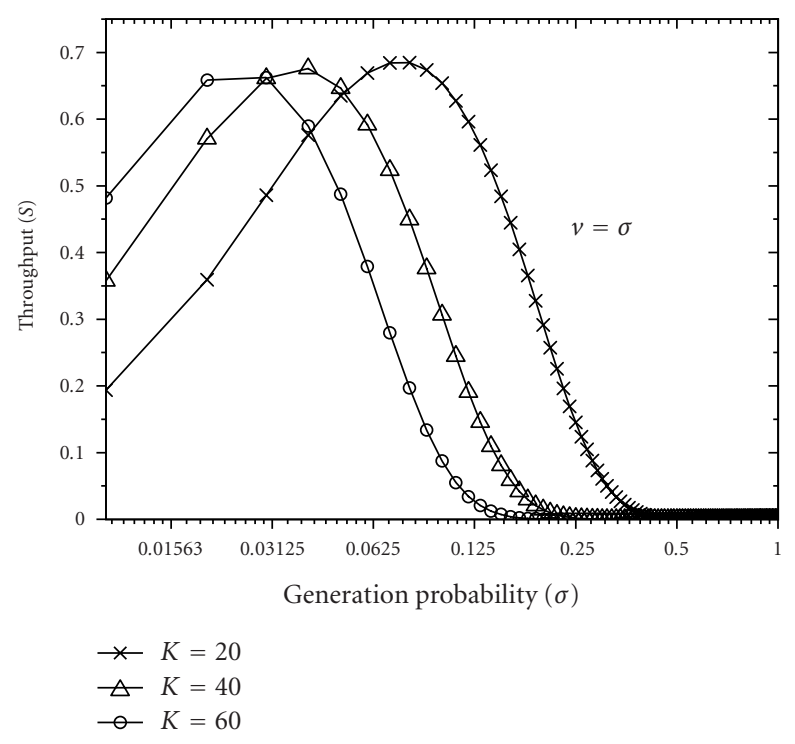

FIgURE 3: Throughput versus generation probability in the proposed scheme with $K$ users.

proposed protocol achieves much higher throughput than the conventional ones. In addition, the achieved throughput approaches to the known highest throughput 0.693 in SICTA whereas the proposed protocol can behave as simple as slotted ALOHA.

We also make the network simulation for the proposed scheme used by a group of $K(K=20,40,60)$ users each with single buffer. At each slot of the simulating system, if the user has no packet in his buffer, he independently generates a packet with probability $\sigma$. If a packet transmission is unsuccessful, the packet is backlogged in the buffer and its retransmission is independently scheduled with probability $v$ except $C$-Slot, where $v=\sigma$. Figure 3 shows the throughput versus generation probability with different number of users. In Figure 3, the highest values of each throughput curve are closed to 0.669 , and hence corroborate the theoretical analysis.

\section{Conclusion}

We have proposed a high-throughput random access protocol for the $2 \times 2$ multiuser MIMO systems. The throughput can be as high as 0.669 per spatial degree of freedom. Our scheme can be readily extended to the $2 \times M(M>2)$ multiuser MIMO systems to accommodate $M$-fold collision [2].

\section{Acknowledgments}

The authors would like to thank the anonymous reviewers for their helpful comments, the research grants are from the Natural Science Foundation of Shanghai (no. 07ZR14104).

\section{References}

[1] G. J. Foschini, "Layered space-time architecture for wireless communication in a fading environment when using multielement antennas," Bell Labs Technical Journal, vol. 1, no. 2, pp. 41-59, 1996.

[2] A. Stamoulis, N. Al-Dhahir, and A. R. Calderbank, "Further results on interference cancellation and space-time block codes," in Proceedings of the 35th Asilomar Conference on Signals, Systems and Computers (ACSSC '01), vol. 1, pp. 257-261, Pacific Grove, Calif, USA, November 2001.

[3] M. K. Tsatsanis, R. Zhang, and S. Banerjee, "Network-assisted diversity for random access wireless networks," IEEE Transactions on Signal Processing, vol. 48, no. 3, pp. 702-711, 2000.

[4] Y. Yu and G. B. Giannakis, "High-throughput random access using successive interference cancellation in a tree algorithm," IEEE Transactions on Information Theory, vol. 53, no. 12, pp. 4628-4639, 2007.

[5] R. Rom and M. Sidi, Multiple Access Protocols: Performance and Analysis, Springer, Berlin, Germany, 1990. 

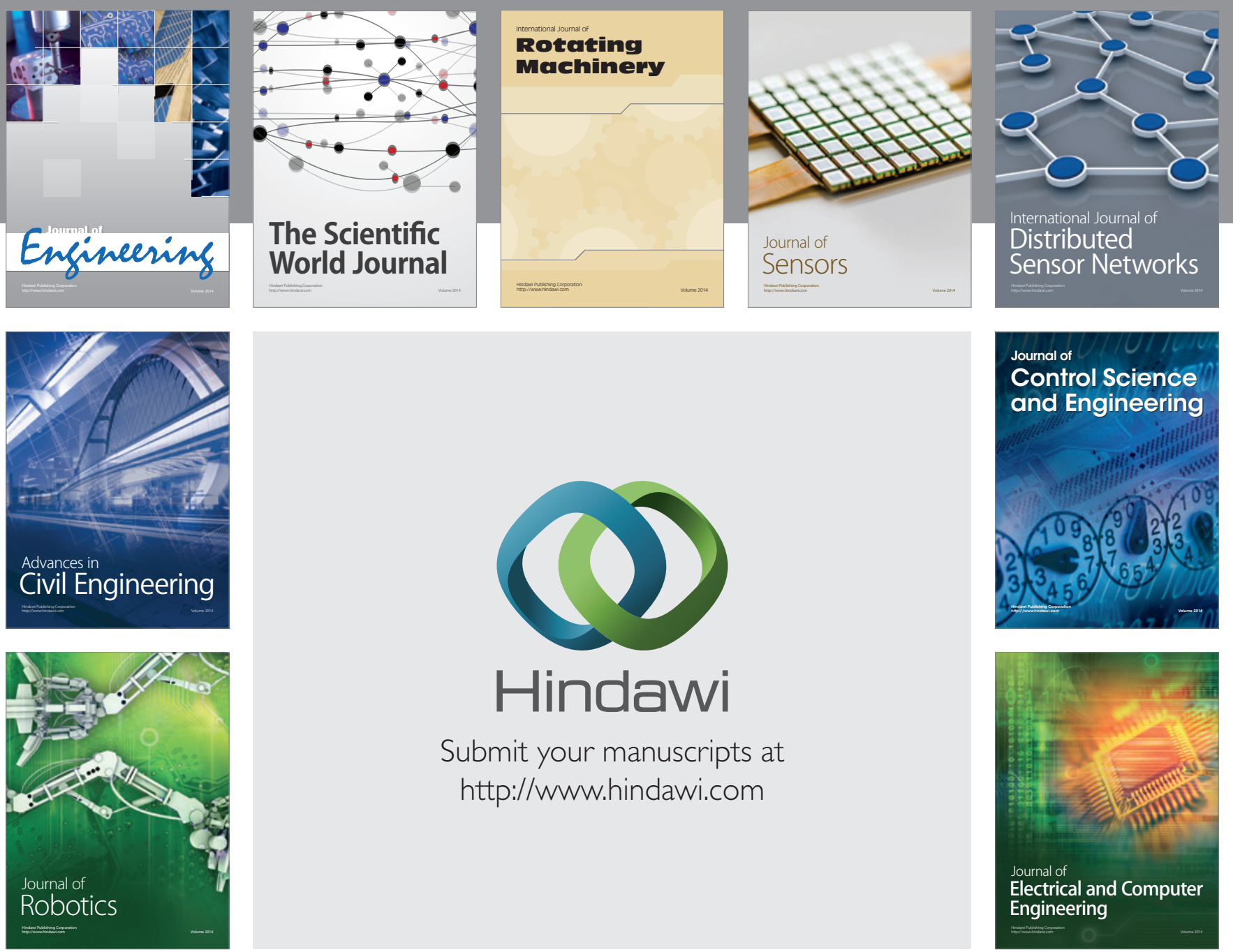

Submit your manuscripts at

http://www.hindawi.com
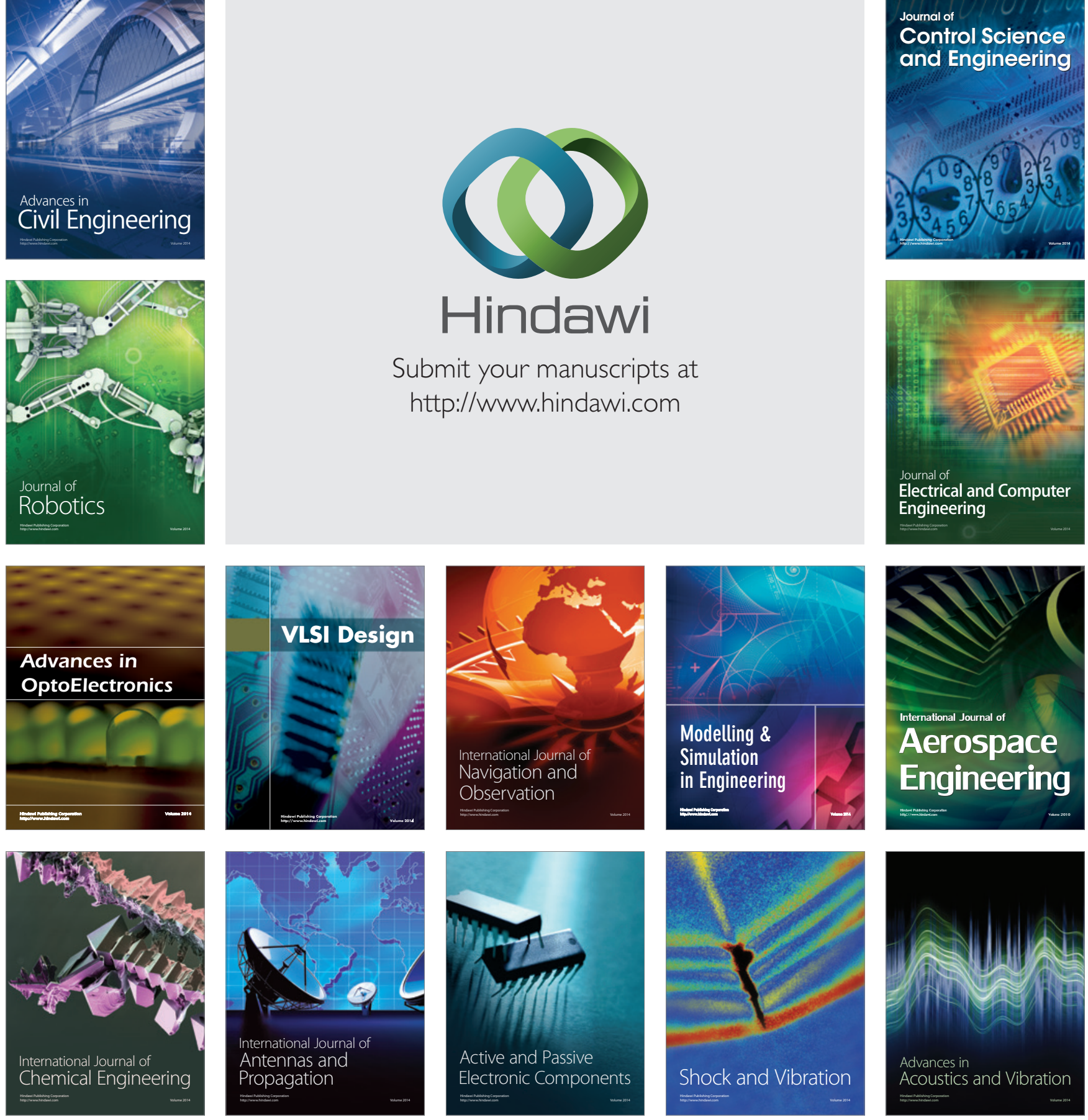